\title{
NONLINEAR IDENTIFICATION OF POWER ELECTRONIC SYSTEMS
}

\author{
K.T. Chau ${ }^{\dagger}$ and C.C. Chan ${ }^{\ddagger}$ \\ ${ }^{\dagger}$ Department of Electrical Engineering \\ Hong Kong Polytechnic, Hong Kong \\ * Department of Electrical and Electronic Engineering \\ The University of Hong Kong, Hong Kong
}

\begin{abstract}
This paper presents a new approach to modelling power electronic systems using nonlinear system identification. By employing the nonlinear autoregressive moving average with exogenous input (NARMAX) technique, the parametric model of power electronic systems can be derived from the timedomain data. This approach possesses some advantages over available circuit-oriented modelling approaches, such as no small-signal approximation, no circuit idealization and no detailed knowledge of system operation. Moreover, it is found that the inclusion of nonlinear terms in the model of power electronic systems is particularly necessary during the presence of large-signal perturbation.
\end{abstract}

\section{INTRODUCTION}

Owing to the switching nature of power electronic systems, the dynamic behaviour is generally nonlinear. Starting from the $1970 \mathrm{~s}$, many researchers have concentrated on obtaining the dynamic model of power electronic systems using small-signal analysis [1]. However, the small-signal modelling can neither handle large-signal perturbations, nor predict harmonic and subharmonic frequency components [2]. Until recently, some nonlinear modelling approaches to power electronic systems have been proposed [3]. Nevertheless, all of them are circuit-oriented, implying that circuit idealization and detailed knowledge of system operation are required. Instead of using circuitoriented modelling approaches, the dynamic behaviour of power electronic systems can be modelled using system identification. Recently, linear system identification has been used to obtain the small-signal model of power electronic systems [4], [5]. However, these linear system identification approaches are illsuited for power electronic systems in the presence of large-signal perturbations. It is the purpose of this paper to develop a new large-signal modelling approach for power electronic systems using nonlinear system identification.

\section{SYSTEM IDENTIFICATION}

System identification deals with the problem of building mathematical models of dynamic systems based on observed data. This area has matured into an established collection of basic techniques that are well understood and known to perform successfully in practical applications [6]. The basic theory of system identification, which is used in this paper, is briefly described to make it self-contained.

In general, the input-output configuration of a system can be described as

$$
\begin{gathered}
y(t)=G(q) u(t)+H(q) e(t) \\
G(q)=\sum_{k=1}^{\infty} g(k) q^{-k} \\
H(q)=1+\sum_{k=1}^{\infty} h(k) q^{-k}
\end{gathered}
$$

where $u(t), y(t)$ and $e(t)$ are the input, output and unmeasurable noise of the system. The functions $G(q)$ and $H(q)$ are to be parameterized, most often as rational functions in the delay operator $q^{-1}$. Thus, this system description can be written as

$$
\begin{gathered}
A(q) y(t)=\frac{B(q)}{F(q)} u(t-n k)+\frac{C(q)}{D(q)} e(t) \\
A(q)=1+a_{1} q^{-1}+\cdots+a_{n a} q^{-n a} \\
B(q)=b_{1}+b_{2} q^{-1}+\cdots+b_{n b} q^{-n b+1} \\
C(q)=1+c_{1} q^{-1}+\cdots+c_{n c} q^{-m c} \\
D(q)=1+d_{1} q^{-1}+\cdots+d_{n d} q^{-n d}
\end{gathered}
$$




$$
F(q)=1+f_{1} q^{-1}+\cdots+f_{n f} q^{-n f}
$$

where $n a, n b, n c, n d$ and $n f$ are the orders of the respective polynomials, and $n k$ is the number of delays from input to output.

The model structure in (4) is too general for most practical purposes. When $n d=n f=0$, the corresponding model is so-called the autoregressive moving average with exogenous input (ARMAX) realization, which has become a standard tool for linear system identification.

The nonlinear autoregressive moving average with exogenous input (NARMAX) model for single-input single-output systems takes the form

$$
\begin{aligned}
y(k)= & \alpha+F^{l}\left[y(k-1), \cdots, y\left(k-n_{y}\right) u(k-d), \cdots, u\left(k-n_{\mu}\right),\right. \\
& \left.\epsilon(k-1), \cdots, \epsilon\left(k-n_{e}\right)\right]+\epsilon(k)
\end{aligned}
$$

where $y(k), u(k)$ and $\epsilon(k)$ are respectively the sampled output, input and prediction error sequences, $d$ is the system time delay, $l$ is the degree of nonlinearity, $\alpha$ is a constant term and $F$ is a nonlinear function [7], [8].

Detecting which terms are significant and should be included in the model is vitally important. Although many structure detection and parameter estimation algorithms have been developed, the orthogonal estimator is adopted because it is both the simplest to implement and to use. By using the regression equation, the NARMAX model can be expressed as

$$
y(k)=\sum_{i=1}^{n_{0}} p_{i}(k) \theta_{i}+\epsilon(k)
$$

where $p_{i}(t)$ represents a term in the NARMAX and no two terms are identical. Rather than directly estimating the parameters $\theta_{i}$ directly, the orthogonal algorithm operates on an equivalent auxiliary model

$$
y(k)=\sum_{i=1}^{n_{\theta}} g_{i} w_{i}(k)+\epsilon(k)
$$

The orthogonal estimator is implemented as follows:

(a) set $w_{l}(t)=p_{I}(t)$,

$$
\hat{g}_{1}=\frac{\sum_{k=1}^{N} w_{1}(k) y(k)}{\sum_{k=1}^{N} w_{1}^{2}(k)}
$$

(b) start from $j=2$ and compute until $j=n_{\theta}$,

$$
\begin{gathered}
\alpha_{i j}=\frac{\sum_{k=1}^{N} w_{i}(k) p_{j}(k)}{\sum_{k=1}^{N} w_{i}^{2}} \quad i=1,2, \cdots, j-1 \\
w_{j}(k)=p_{j}(k)-\sum_{i=1}^{j-1} \alpha_{i j} w_{i}(k) \\
\hat{g}_{j}=\frac{\sum_{k=1}^{N} w_{j}(k) y(k)}{\sum_{k=1}^{N} w_{j}^{2}(k)}
\end{gathered}
$$

(c) compute the NARMAX parameters backwards,

$$
\hat{\theta}_{n_{b}}=\hat{\boldsymbol{g}}_{n_{\theta}}
$$

$$
\hat{\theta}_{i}=g_{i}-\sum_{j=i+1}^{n_{0}} \alpha_{i j} \hat{\theta}_{j} \quad i=n_{\theta}-1, n_{\theta}-2, \cdots, 1
$$

where $N$ represents the number of data points. The auxiliary regressors $w_{i}(t)$ are orthogonal so that additional terms can be added to the model without the need to recompute all the previous $\hat{g}_{j}$, $j<i$. In practice, the prediction errors are estimated as

$$
\hat{\epsilon}(k)=y(k)-\sum_{i=1}^{n_{0}} g_{i} w_{i}(k)
$$

By estimating all the process parameter terms first, then computing the prediction errors and estimating the noise model, the orthogonal algorithm provides an efficient method of estimating all the unknown coefficients in the NARMAX model.

Increasingly, in order to detect which terms should be included in the model, the error reduction ratio is computed as

$$
E R R_{i}=\frac{\hat{g}_{i}^{2} \sum_{k=1}^{N} w_{i}^{2}(k)}{\sum_{k=1}^{N} y^{2}(k)} \times 100
$$

which provides a measure of the reduction in mean squared error resulted by including the $i$ th term in the 
NARMAX model. Usually, the $i$ th term is included if $E R R_{i}$ exceeds the thresholds of $C_{d}$ and $C_{d e}$ for the process and noise terms respectively. It should be noted that the value of $E R R_{i}$ may be affected by the position of $p_{i}(k)$.

This difficulty can be overcome by using the forward regression version of the aforementioned algorithm. Firstly, calculate

$$
\begin{gathered}
w_{1}^{(i)}(k)=p_{i}(k) \\
\hat{g}_{1}^{(i)}=\frac{\sum_{k=1}^{N} w_{1}^{(i)}(k) y(k)}{\sum_{k=1}^{N}\left(w_{1}^{(i)}(k)\right)^{2}} \\
{[E R R]_{1}^{(i)}=\frac{\left(\hat{g}_{1}^{(i)}\right)^{2} \sum_{k=1}^{N}\left(w_{1}^{(i)}(k)\right)^{2}}{\sum_{k=1}^{N} y^{2}(k)}}
\end{gathered}
$$

and find the maximum of $[E R R]_{I}^{(i)}$, say $\left[E R R J_{I}^{(j)}\right.$. Then the first term to include in the model is selected as $w_{l}(k)=w_{l}^{(j)}(k)$. Secondly, compute

$$
\begin{aligned}
w_{2}^{(i)}(k) & =p_{i}(k)-\alpha_{12}^{(i)} w_{1}(k) \\
\alpha_{12}^{(i)} & =\frac{\sum_{k=1}^{N} w_{1}(k) p_{i}(k)}{\sum_{k=1}^{N} w_{1}^{2}(k)} \\
\hat{g}_{2}^{(i)} & =\frac{\sum_{k=1}^{N} w_{2}^{(i)}(k) y(k)}{\sum_{k=1}^{N}\left(w_{2}^{(i)}(k)\right)^{2}} \\
{[E R R]_{2}^{(i)} } & =\frac{\left(\hat{g}_{2}^{(i)}\right)^{2} \sum_{k=1}^{N}\left(w_{2}^{(i)}(k)\right)^{2}}{\sum_{k=1}^{N} y^{2}(k)}
\end{aligned}
$$

and find the maximum of $[E R R]_{2}^{(i)}$, say $[E R R]_{2}^{(i)}$. Then the second term $w_{2}(k)=w_{2}^{(t)}(k)$ is selected. This procedure is terminated when at any step $q$ say

$$
[E R R]_{g}<C_{d} \text { or } C_{d e}
$$

or when the total parameter set has been searched.

Model validation is to test whether the model structure and parameter estimates are correct. If any significant terms have been omitted from the model or any parameters incorrectly estimated, $\epsilon(k)$ will not satisfy the following tests:

$$
\begin{array}{ll}
\Psi_{\epsilon \epsilon}(\tau)=\delta(\tau) \\
\Psi_{u \epsilon}(\tau)=0 & \forall \tau \\
\Psi_{u^{2^{\prime} \epsilon}}(\tau)=0 & \forall \tau \\
\Psi_{u^{2^{\prime} \epsilon^{2}}}(\tau)=0 & \forall \tau \\
\Psi_{\epsilon \epsilon u}(\tau)=0 & \forall \tau \geq 0
\end{array}
$$

where the dash indicates that the mean has been removed. Thus, the orthogonal algorithm should be reentered to investigate the deficiency.

\section{TIME-DOMAIN SIMULATION}

SPICE, or its PC version PSpice, is one of the most popular circuit simulators [9]. It has been accepted by designers and researchers to perform realistic simulation of both analogue and digital electronic systems. Its special feature is the availability of realistic models of most electronic components. Thus, the resulting simulation is not a theoretical analysis, but a practical prediction. Instead of building experimental prototypes, SPICE or PSpice simulation is usually adopted to verify the theoretical result.

Recently, many researchers have focused on extending the applicability of SPICE simulation to power electronics. Although the use of SPICE for power electronic systems still suffers from the problem of lengthy simulation, it is an economical and reliable simulator to study the time-domain behaviour of power electronic systems [10].

One of the most popular power electronic systems, the DC-DC converter, is used for exemplification. This power converter consists of a DC voltage source $V_{s}$, a power MOSFET, a power diode, an inductor $L$ with an equivalent series resistor $r_{L}$, a capacitor $C$ with an equivalent series resistor $r_{C}$ and an output resistor $R$. The output voltage is controlled by adjusting the turn-on period of the power MOSFET, so-called the duty-cycle control or pulsewidth modulation (PWM). This control signal is the output of a comparator, 
which is produced by comparing the ramp generator's sawtooth signal and the duty cycle. Thus, a random duty-cycle signal is purposely injected into the system. This random signal is uniformly distributed in such a way that system dynamics of the converter can be fully excited. Initially, both inductor current $i_{L}$ and capacitor voltage $v_{c}$ are zero.

Although various realistic models of voltage sources, power MOSFETs, power diodes, inductors, capacitors and resistors have been available in the library, SPICE or PSpice does not have a built-in way to generate a random signal in the time domain. However, the random signal can be simulated by using its piecewise linear (PWL) voltage source model. This PWL source model, which consists of a list of random numbers at specific instants of time, can be easily created using the random number generator available in other software packages such as MatLab and MathCAD.

During the time-domain simulation, the duty cycle and output voltage are recorded according to the predefined sampling interval. The resulting database is then stored as table form in the output file for parametric identification. The data set used for identification should be different from that for verification. Otherwise, the fit always improves with the increase of model structure order.

It should be noted that the input excitation is the duty cycle when the control-to-output response is required. In case of the investigation of the line-tooutput response, the input becomes the DC voltage source.

\section{APPLICATION EXAMPLES}

The NARMAX approach to modelling power electronic systems is exemplified using two basic PWM DC-DC converter - boost and buck. The input is equivalent to the converter duty cycle while the output is the load voltage.

\section{A. PWM Boost Converter}

The circuit diagram of a basic PWM boost converter is shown in Fig. 1. The duty-cycle signal is uniformly distributed in the operating range from 0.35 to $\mathbf{0 . 6 5}$.

The corresponding loss functions of the NARMAX model without nonlinear terms, linear identification, are tabulated in Table I. It can be found that the use of order 2 and delay 0 for the model is appropriate. However, inspection of the model validity tests shows that nonlinear terms are missing from the model and the parameter estimates are biased. As shown in Fig. 2 , the predicted output obtained from using the linear model is compared with the actual output. The discrepancy is due to the system nonlinearity. Based on this linear model, the degree of nonlinearity is initially set to 2 which is a good starting point for the nonlinear search. By employing the forward regression estimator to test the significance of nonlinear terms, the resulting significant nonlinear terms are $u(t)^{2}$, $u(t-1)^{2}, y(t-1)^{2}$ and $y(t-1) u(t-1)$. Therefore, the NARMAX model of the boost converter is given by

$$
\begin{aligned}
y(t)= & 13.08+58.76 u(t)-36.73 u(t-1) \\
& +0.1823 y(t-1)-0.034 y(t-2) \\
& -40.72 u(t)^{2}+28.76 u(t-1)^{2} \\
& -0.0012 y(t-1)^{2}+1.0419 y(t-1) u(t-1)
\end{aligned}
$$

By using this NARMAX model, the predicted output is compared with the actual output in Fig 3. A good agreement can be found. To further testify the resulting model, a sinusoidal wave with dc offset is injected into the converter via the duty-cycle control input. The actual output obtained by PSpice simulation is compared with the predicted outputs obtained from the linear and NARMAX models, respectively shown in Figs. 4 and 5. As expected, the NARMAX model is much better than the linear model. It should be noted that the advantage of NARMAX model is due to the inclusion of nonlinear terms. In particular, when the input signal perturbation is large, the system nonlinearity becomes remarkable.

\section{B. PWM Buck Converter}

By including parasitic resistances, a realistic PWM buck converter is shown in Fig. 6. The duty-cycle signal is uniformly distributed in the whole operating range from 0 to 1 . Inspection of Table II shows that the use of order 2 and delay 0 is an appropriate selection. Again, model validity tests show that nonlinear terms are missing. By setting the degree of nonlinearity of the NARMAX model to 2 and searching for significant nonlinear terms using the forward regressive algorithm, it can be found that only one nonlinear term $u(t)^{2}$ should be included in the model. As shown in Fig. 7, the resulting predicted output closely agrees with the actual output. Moreover, by injecting a sinusoidal wave with $\mathrm{dc}$ offset into the system, the PSpice simulated output has a significant discrepancy with the predicted output obtained from the linear model as shown in Fig. 8. As expected and shown in Fig. 9, the predicted output from the NARMAX model closely agrees with the actual output. 


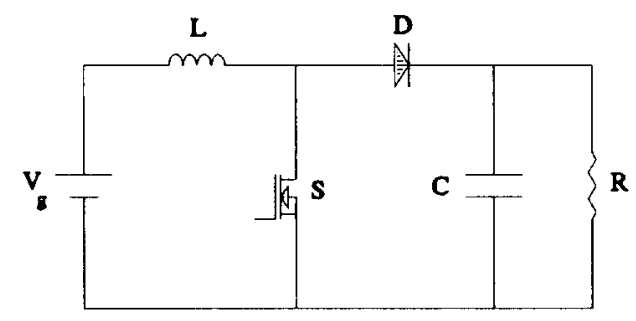

Fig. 1. PWM boost converter.

Table I. Loss functions of PWM boost converter.

\begin{tabular}{c|ccc}
\hline & \multicolumn{3}{|c}{ Delay } \\
Order & 0 & 1 & 2 \\
\hline 1 & 935.1 & $3.1 \times 10^{3}$ & $1.5 \times 10^{3}$ \\
2 & 34.6 & $2.7 \times 10^{3}$ & $7.2 \times 10^{3}$ \\
3 & 27.2 & $2.6 \times 10^{32}$ & $6.2 \times 10^{3}$ \\
\hline
\end{tabular}

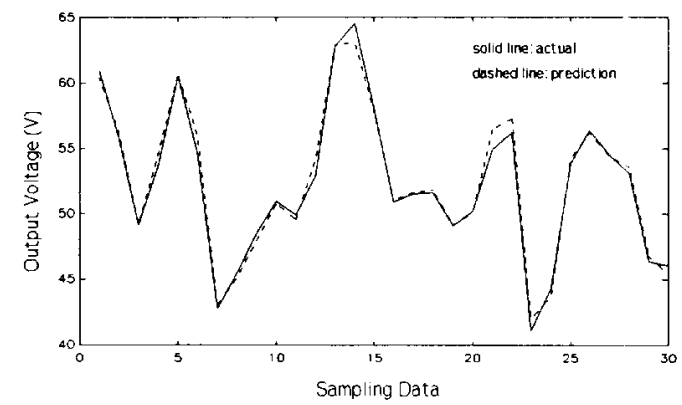

Fig. 2. Random outputs of boost by linear id.

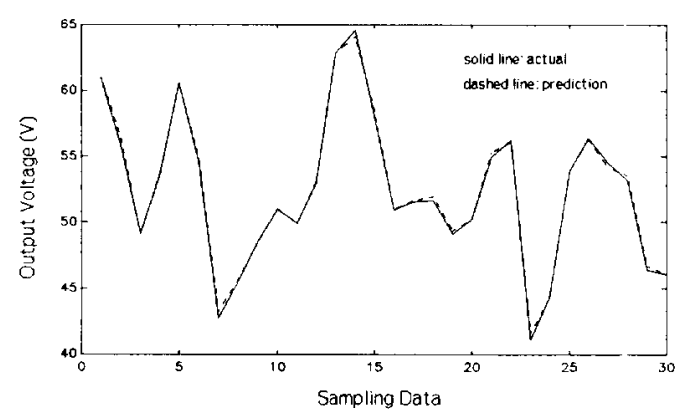

Fig. 3. Random outputs of boost by nonlinear id.

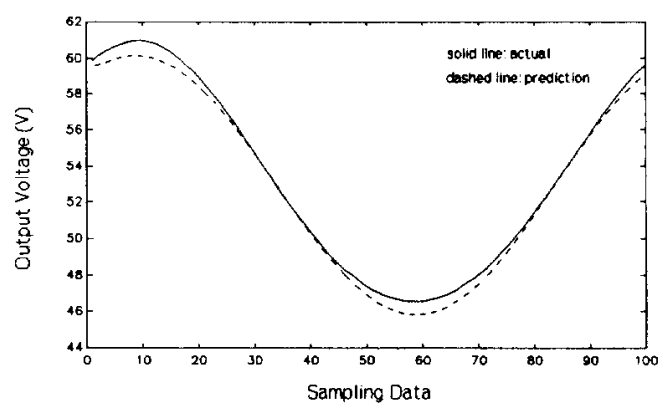

Fig. 4. Sinusoidal outputs of boost by linear id.

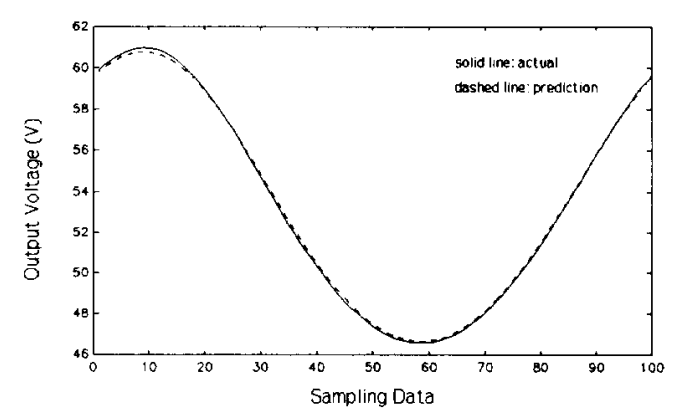

Fig. 5. Sinusoidal outputs of boost by nonlinear id.

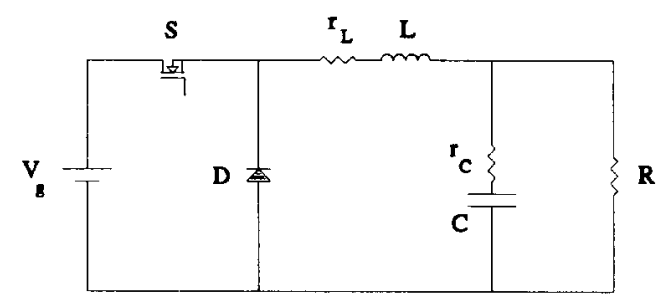

Fig. 6. PWM buck converter.

Table II. Loss functions of PWM buck converter.

\begin{tabular}{c|ccc}
\hline & \multicolumn{3}{|c}{ Delay } \\
Order & 0 & 1 & 2 \\
\hline 1 & 55.35 & 971.1 & 643 \\
2 & 4.615 & 370.5 & 637.6 \\
3 & 4.366 & $8.1 \times 10^{32}$ & 109.4 \\
\hline
\end{tabular}




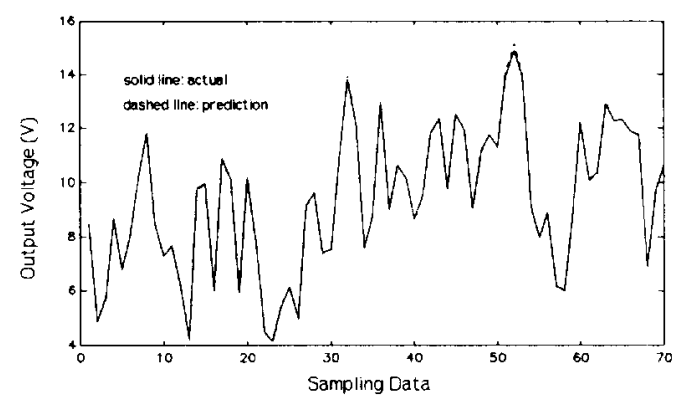

Fig. 7. Random outputs of buck by nonlinear id.

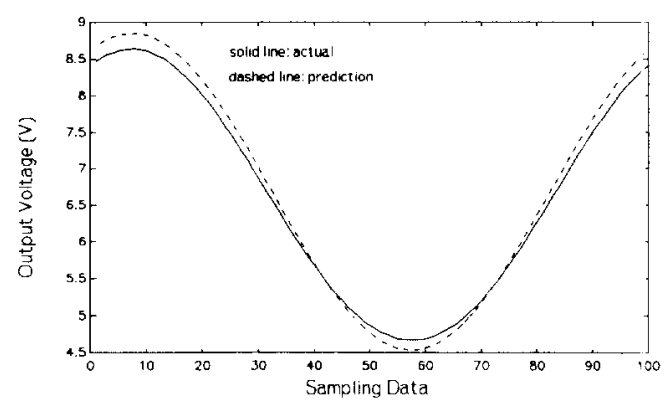

Fig. 8. Sinusoidal outputs of buck by linear id.

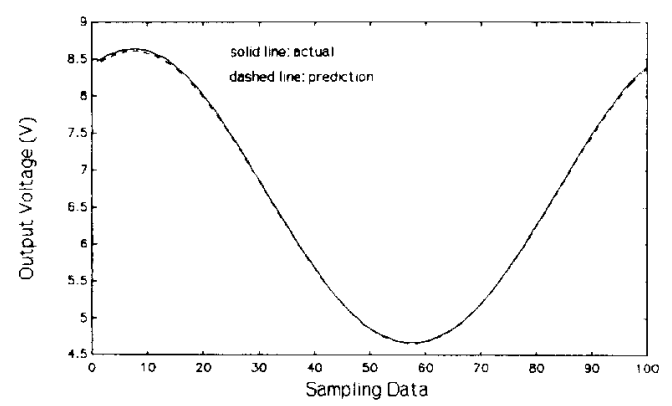

Fig. 9. Sinusoidal voltages of buck by nonlinear id.

\section{CONCLUSION}

A new computer-aided modelling approach for power electronic systems using NARMAX has been presented. It has also been verified that the inclusion of nonlinear terms is necessary for the modelling of power electronic systems in the presence of largesignal perturbation. As compared with available circuit-oriented modelling approaches, this approach possesses the advantages of no small-signal approximation, no circuit idealization and no detailed knowledge of system operation.

\section{ACKNOWLEDGMENT}

The authors gratefully acknowledge the contribution of Mr. C.C. Cheng, and the support in part of Hong Kong Polytechnic.

\section{REFERENCES}

[1] K.T. Chau, Y.S. Lee and A. Ioinovici, "Computer-aided modeling of quasi-resonant converters in the presence of parasitic losses by using MISSCO concept," IEEE Trans. Ind. Electron., vol. 38, pp. 455-462, 1991.

[2] C.C. Chan and K.T. Chau, "Nonlinear modeling of pulsewidth-modulated and quasiresonant converters," in Proc. Int. Conf. Ind. Electron. Contr. Instru., 1993, pp. 361-374.

[3] C.C. Chan and K.T. Chau, "Spectral modeling of switched-mode power converters," IEEE Trans. Ind. Electron., vol. 41, pp. 441-450, 1994.

[4] P. Maranesi, "Small-signal circuit modeling in the frequency-domain by computer-aided timedomain simulation," IEEE Trans. Power Electron., vol. 7, pp. 83-88, 1992.

[5] K.T. Chau and C.T. Tse, "Identification of power electronic systems," in Proc. Int. Power Eng. Conf., 1995.

[6] L. Ljung, System Identification: Theory for the User. Prentice-Hall, 1987.

[7] S. Chen and S.A. Billings, "Representations of nonlinear systems: the NARMAX model," Int. J. Control, vol. 49, 1989, pp. 1013-1032.

[8] S.A. Billings and K.M. Tsang, "Spectral analysis for non-linear systems, part I: parametric non-linear spectral analysis," Mech. Sys. Sig. Process., vol. 3, pp. 319-339, 1989.

[9] PSpice Reference Manual Version 5.0. MicroSim Corporation, Irvine, CA, 1991.

[10] M.H. Rashid, SPICE for Power Electronics and Electric Power. Prentice-Hall, 1993. 